\title{
Visual Similarity Versus Mental Rotation For Inverted and Upright Faces.
}

Sam S. Rakover ( $\square$ rakover@psy.haifa.ac.il )

University of Haifa

Rani A. Bar-On

University of Haifa

Anna Gliklich

University of Haifa

\section{Research Article}

Keywords: Face Inversion Effect (FIE), transformation of inversion, face

Posted Date: February 26th, 2021

DOI: https://doi.org/10.21203/rs.3.rs-223102/v1

License: (1) This work is licensed under a Creative Commons Attribution 4.0 International License. Read Full License 


\section{Abstract}

A major interest of research in face recognition lies in explaining the Face Inversion Effect (FIE), in which the recognition of an inverted face is less successful than that of an upright face. However, prior research has devoted little effort to examining how the cognitive system handles comparison between upright and inverted faces. In two experiments, such comparison is found to be based on visual similarity rather than on mental rotation of the inverted face to upright. Visual similarity is based on certain elements mutual to the two faces, which resist the transformation of inversion. These elements are symmetrical or salient components of the face, such as round eyes or thick lips.

\section{Introduction}

Research on face perception and recognition over the last 50 years has focused on the Face Inversion Effect (FIE), according to which an upright face (with the hair on top and the chin below) is recognized much better than an inverted face (chin on top, hair below) ${ }^{1-7}$. The FIE is explained by two similar hypotheses, 1 ) the processing of configural information related to the space between facial features and 2) holistic perception of the face as a whole. The hypotheses propose respectively that processing of configural or holistic information is impaired in an inverted face compared to an upright face $1,3,4,5,8,9$. Despite the vast amount of research on the FIE, the question of how the cognitive system compares an inverted face to an upright face has been largely neglected. As a possible answer we tested two hypotheses: visual similarity and mental rotation.

The visual-similarity hypothesis proposes that a person's decision is based on similarity of mutual alike elements between the perceived inverted face and the remembered upright face $\mathrm{e}^{10-12}$. The mental-rotation hypothesis proposes that the inverted face as a whole is mentally rotated to the upright orientation and then compared to the remembered upright face ${ }^{13-16}$. While the FIE can be explained by the great difficulty of mentally rotating each of the facial features to the upright orientation ${ }^{14,15}$, empirical evidence supports the hypothesis that a face is mentally rotated as a whole unit, similar to the mental rotation of entire visual shapes ${ }^{16-18}$.

These two hypotheses do not overlap since they stem from two distinct mechanisms. While the visualsimilarity hypothesis is based mainly on the estimation of the number of mutual elements that compound the two faces ${ }^{10-12}$, the mental-rotation hypothesis is founded on a mechanism that rotates the representation of the inverted face to the upright orientation and then examines whether it overlaps with the remembered upright face ${ }^{13-18}$.

The main goal of the present paper is to empirically test these two hypotheses. We conducted two experiments based on the following manipulations and rationale. First, in a preparatory experiment, we constructed two groups of faces. The similargroup contained seven different pairs, each composed of two different faces that had obtained high similarity index, one upright and one inverted. The non-similar 
group contained seven different pairs each composed of two different faces, one upright and one inverted, which had obtained low similarity index.

Secondly, in a prediction testing experiment, a variation of the Yes/No procedure, we presented to the participants in a Study stage, 14 upright faces taken from both the similarand non-similargroups. In a subsequent Testing stage, 28 inverted faces were presented. They were composed of the 14 previously viewed upright faces (of the Study stage) and 14 new faces. The 14 new faces included seven inverted faces from the similargroup and seven from the non-similar group. The participant's task was to decide for each inverted face if it was old (familiar) or new.

If the visual-similarity hypothesis is correct, then we can predict that the false-alarm measure for the seven new, similar, inverted faces (FAs-faces) will be significantly greater than the false-alarm for the seven new, non-similar, inverted faces (FAns-faces). When the visual similarity between upright face and inverted face is high, there is a greater likelihood of believing that a new face is an old one; hence, FAsfaces $>$ FAns-faces.

In contrast, if the mental-rotation hypothesis is correct, there should be no significant differences between FAs-faces and FAns-faces; when an inverted face is rotated to the upright position, it should be easy to decide whether or not it is congruent with the remembered upright face and therefore to decide whether the inverted face is old or new.

To test these two predictions, we conducted two experiments. The goal of the preparatory Experiment 1 was to construct two groups of faces: similar and non-similar. The testing Experiment 2 had two parts. The first, Similarity, was to test the above two predictions empirically, and the second, Orientation, had two important goals. First, to give additional empirical support to the construction of the similarity groups by using the technique of ranking and second, to test whether the distinction between the similar and the non-similar groups was confined only to the pair of faces in the Upright/Inverted (UI) orientations or if it could be generalized to the other orientations, UU, IU and II. In other words, Part B of Experiment 2 was to test whether this distinction has the quality of resisting the inversion transformations.

\section{Experiment 1 - Preparatory Study}

Methods

The study has been reviewed and approved by the Psychology department ethic committee and the University of Haifa's IRB (application no. 234/17). All methods were carried out in accordance with relevant guidelines and regulations. The materials and data are available at https://drive.google.com/drive/folders/1Vxo48qsm742JVGE1H_TWBKV0K9A7Lt7s?usp=sharing.

The black and white face-pictures used in the present study, were taken from 2 publicly available online data banks back in the 1990's. These pictures were used in several previous experiments and they are not of the participants of the present or of previous experiments. 
Participants, Design, and Procedure: Thirty participants (21 females and nine males, average age 24.7) were shown a series of face pictures on a computer screen. The number of participants was determined on the basis of a pilot study and previous experience with similar kind of experiments. The participants were undergraduate students who were rewarded by payment or course credit. Informed consent was obtained for the experiments reported here. In each trial, participants were presented with six oval shaped faces without hair or ears. One of the faces appeared in the upright orientation (hair on top, chin below) and the other five appeared in the inverted orientation (chin on top, hair below) and were arranged in a semi-circle below the upright face. Each oval face was a different unfamiliar, black-and-white image of a man. The hair and ears were cut because external facial features would aid recognition ${ }^{19,20}$.

A total of 180 trials included 30 different faces appearing six times each in the upright orientation with five different inverted faces below. Thus, each upright face was associated with all 30 faces in the inverted orientation (including itself). The faces, chosen randomly for each trial, were exposed for 20 seconds, in which time the participant had to choose among the five inverted faces at least one similar to the upright face. Each trial also featured two whistles, at 10 and 18 seconds, to expedite the participant's responses.

Results

The main purpose of the preparatory Experiment 1 was to construct two groups of face pairs: similar and non-similar. To do this, we constructed a table of 30 upright faces $\times 30$ inverted faces. The table has $30 \times 30=900$ cells. Each cell shows how many of the 30 participants indicated that a given inverted face was similar to a certain upright face. For example, cell ${ }_{4,15}=15$ means that half of the participants indicated that inverted face ${ }_{4}$ is similar to upright face ${ }_{15}$. Based on this result, we built a similar group by selecting seven different pairs of different faces (upright and inverted) with high similarity index (similarity was in the range between $27 \%$ and $67 \%$ of the participants). The non-similar group was constructed in the same way; we selected seven different pairs of different faces (upright and inverted) with low similarity index (similarity was in the range between $3 \%$ and $17 \%$ of the participants). The pairs in these two groups were different from each other. Figure 1 shows examples of the similarand nonsimilargroups.

It was found that on average, $82 \%$ of participants indicated that an inverted face was similar to itself in the upright orientation. In comparison, an average of $23 \%$ of participants indicated that a certain inverted face was similar to a different upright face. With regard to similarity, this finding suggests that the number of elements mutual to upright face $X$ and inverted face $X$ is greater than the number of elements mutual to upright face $X$ and inverted face $Y$.

\section{Experiment 2 - Prediction Testing}

Methods

Part A - Similarity 
Participants, Design, and Procedure: Twenty participants (15 females and five males, average age 24.75) were shown a series of pictures on a computer screen each consisting of one face at a time. The experiment consisted of two stages: Study and Test and was a variation of the common Yes/No recognition experiment. In the Study stage, participants were shown 14 upright faces, each exposed for 3s at intervals of $1 \mathrm{~s}$ between faces. These upright faces were taken from both the similar and non-similar groups and presented in randomized order. In the Test stage, participants were shown 28 inverted faces including 14 old faces that appeared in the Study stage and 14 new faces taken from both the similar and non-similar groups. The faces in this stage were also presented in randomized order.

The participants were informed that they would take part in two experiments in succession. The instructions for each experiment were read before the beginning of each experiment. For Part A, the Study stage, participants were told to concentrate on the upright oval-faces and attempt to remember them. In the Test stage, they were presented with old and new inverted faces, one at a time, and had to decide whether they were old or new. They had 10 s to make their decision. After $5 \mathrm{~s}$, a whistle sounded to expedite their decision.

\section{Part B - Orientation}

Participants, Design \& Procedure: The same 20 participants were shown a series of pairs of faces, one at a time, on a computer screen. All the pairs were constructed of the14 pairs constructed in the preparatory study, Experiment 1. Each face pair was presented in four possible orientations: upright/inverted (UI), upright/upright (UU), inverted/inverted (II) and inverted/upright (IU). A total of 56 face pairs (4x14) were presented one at a time in randomized order. Each pair was presented for 10 s to allow the participant to rank the degree of similarity between the faces on the screen; a score of 1 signifies that the faces were not similar at all, while a 5 signifies that the faces were very similar. After $5 \mathrm{~s}$, a whistle sounded to indicate that half the time remained.

Results

Part $A$ The main results appear in Figure 2. While there is no significant difference between Percent Hits in the similargroups (\%Hs) and Percent Hits in the non-similargroups (\%Hns), Percent False-Alarm in the similar-groups (\%FAs) is significantly greater than Percent False-Alarm in the non-similar-groups (\%FAns). A repeated measurement 2 (Hits, False-Alarm) $\times 2$ (similar group, non-similargroup) ANOVA supports this observation: $F(1,19)=9.56 p<.006 \mu^{2}=.34 ; A$ LSD test revealed a significant difference between $\% F A s=55.0 \%$ and $\% F A n s=35.7 \% \mathrm{p}<.007$ but not between $\% \mathrm{Hs}$ and $\% \mathrm{Hns}$.

Part $B$ The main results appear in Figure 3. The similarity ranking of the pairs in the similar group (SRs) was higher than the similarity ranking in the non-similargroup (SRns) in all the four groups: UI, UU, IU and II. A repeated measurement $4(\mathrm{UU}, \mathrm{UI}, \mathrm{IU}, \mathrm{II}) \times 2$ (Similar group, Non-similar group) ANOVA supports this observation: $F(3,57)=27.92 p<.0001 \mu^{2}=.595$. A LSD test done within each of the four groups revealed that SRs was significantly higher than SRns at the level of $p<.001$. 


\section{Discussion}

The main results of the present study are as follows: First, \%FAs is greater than \%FAns, while no significant difference was found between \%Hs and \%Hns. Second, the SRs was higher than the SRns in each of the four orientations. The first result supports the visual similarity hypothesis that the cognitive system compares an inverted face with an upright face by conducting a visual similarity between the perceived inverted face and the remembered upright face ${ }^{10,11}$. The result does not support the mental rotation hypothesis since it predicts no significant difference between \%FAs and \%FAns.

Given this, one may propose the combined hypothesis, a kind of a combination of mental rotation and visual similarity; the inverted face is rotated to the upright orientation and then visual similarity is activated. One important argument against this hypothesis is that it involves a huge mental effort; one has to rotate the perceived face to the upright orientation and then to compare it with the remembered upright face to evaluate their visual similarity. Conversely, according to the visual-similarity hypothesis, one can simply evaluate the visual similarity of the two faces (inverted and upright) directly. As can be seen from the results of the Experiment 2, Part B, visual similarity resists the transformation of inversion. As an example, consider the II pair (see Figures 1 and 3). According to the combined hypothesis, the similarity evaluation of the II pair should be done indirectly, after mental rotation of each of the inverted faces to the upright orientation - an action that demands a great mental effort. In contrast, according to the visual-similarity hypothesis, the evaluation is direct and does not demand so much mental effort.

The second result supports the validity of the construction of the similarand non-similargroups; the SRs is greater than the SRns for the UI orientation and also in the other three orientations. Hence, the distinction between the similar and non-similar groups is not confined only to the Ul orientation. Rather, the distinction generalizes to all other orientations. This finding suggests that the similarity between different faces resists the transformation of inversion. If we assume that similarity is founded on mutual elements that resist transformation of inversion, inversion resisting elements (IRE), we may propose that IRE have the qualities of symmetry or saliency, for example, round eyes, fleshy lips, wide nose, and thick eyebrows. This explains well the above finding; the similarity of an upright, oval face with itself in the inverted orientation is much higher than the similarity between an upright face with a different inverted face. The number of mutual symmetrical or salient elements in an upright face $X$ and an inverted face $X$ is much higher than in an upright face $\mathrm{X}$ and an inverted face $\mathrm{Y}$. Furthermore, the IRE may also explain why recognition of inverted faces is based mainly on featural information ${ }^{2,3,12}$. Promising future research would discover which IREs are the most (or least) salient for successful face recognition.

\section{References}

Maurer, D., Le Grand, R., \& Mondloch, C. J. (2002). The many faces of Configural processing. Trends in Cognitive Sciences, 6, 255-260. Rakover, S. S. (2002). Featural vs. configurational information in faces: A conceptual and empirical analysis. British Journal of Psychology, 93, 1-30. Rakover, S. S. (2013). Explaining the face-inversion effect: The face-scheme incompatibility (FSI) model. Psychonomic Bulletin 
\& Review, 20, 665-692. Rossion, B. (2008). Picture-plane inversion leads to qualitative changes of face perception. Acta Psychologica, 128, 274-289. Rossion, B. (2009). Distinguishing the cause and consequence of face inversion: The perceptual field hypothesis. Acta Psychologica, 132, 300-312. Valentine, T. (1988). Upside-down faces: A review of the effect of inversion on face recognition. British Journal of Psychology, 79, 471-491. Yin, R. K. (1969). Looking at upside-down faces. Journal of Experimental Psychology, 81, 141-145. McKone, E. (2010). Face and object recognition: How do they differ? In V. Coltheart (Ed.), Tutorials in visual cognition (pp. 261-303). New York, NY: Psychology Press. Piepers, W., \& Robbins, R. A. (2012). A review and clarification of the terms "holistic," "configural" and "relational" in face perception literature. Frontiers in Psychology, 3, Article 559. Tversky, A. (1977). Features of similarity. Psychological Review, 84, 327-352. Rakover, S. S. \& Cahlon, B. (1989). To catch a thief with a recognition model: The model and some empirical results. Cognitive Psychology, 21, 423-468. Rakover, S. S. \& Cahlon, B. (2001). Face Recognition: Cognitive and Computational Processes. Amsterdam/Philadelphia: John Benjamins. Rakover, Sam S. (2015). Cognitive processing of scrambled faces: Effects of instructions and tasks. American Journal of Psychology, 128, 379-386. Rock, I. (1973). Orientation and form. New York, NY: Academic Press. Rock, I. (1974). The perception of disoriented figures. Scientific American, 230, 78-86. Valentine, T. \& Bruce, V. (1988). Mental rotation of faces. Memory \& Cognition, 16, 556-566. Shepard, R. N. \& Metzler, J. (1971). Mental rotation of three-dimensional objects. Science, 171, 701-703. Cooper, L. A. (1975). Mental rotation of random two-dimensional shapes. Cognitive Psychology, 7, 20-43. Bonner, L., Burton, A. M., \& Bruce, V. (2003). Getting to know you: How we learn new faces. Visual Cognition, 10, 527-536 Want, S. C., Pascalis, O., Coleman, M., \& Blades, M. (2003). Recognizing people from the inner or outer parts of their faces: Developmental data concerning "unfamiliar" faces. British Journal of Developmental Psychology, 21, 125-135.

\section{Figures}




\section{Similar Upright-Inverted Pair}
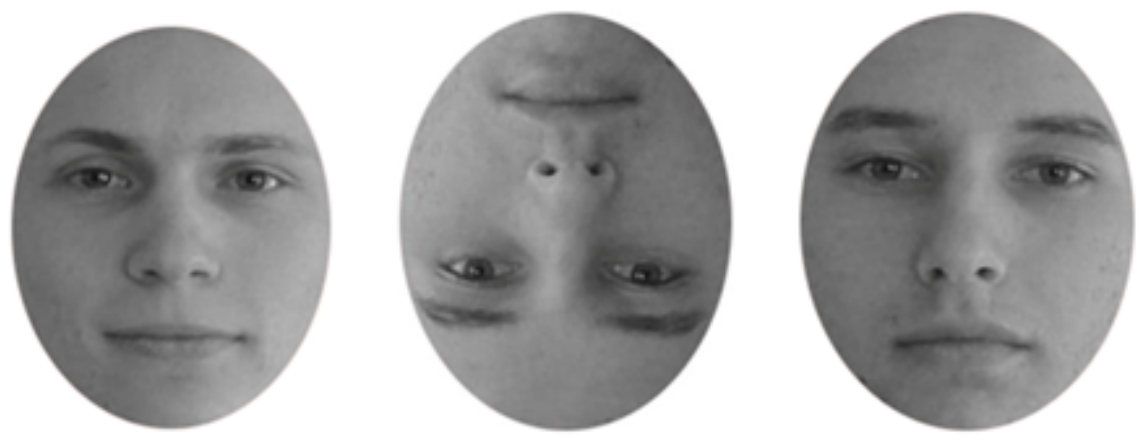

Non-Similar Upright-Inverted Pair
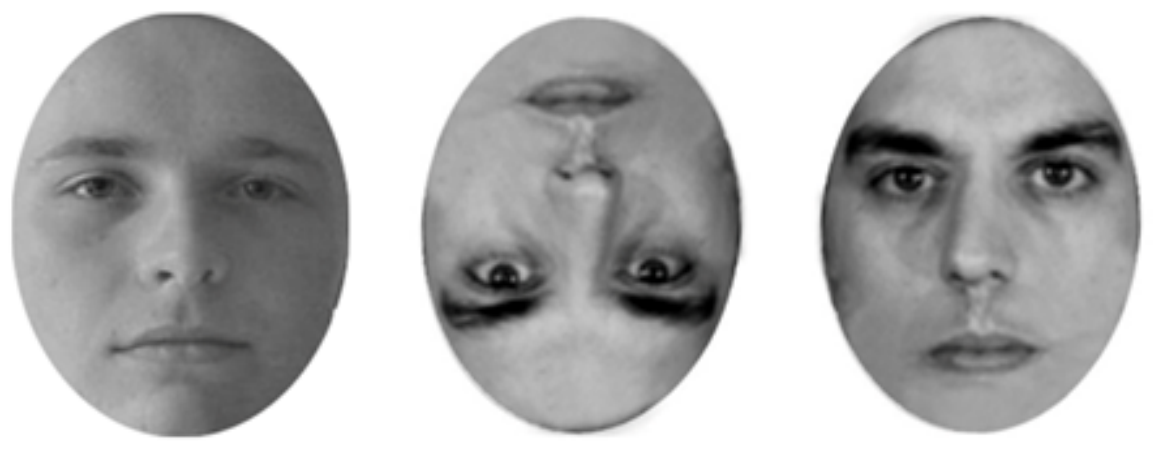

Figure 1

Examples of similar and non-similar pairs of oval-faces. The left face was presented in the upright orientation and the middle one in the inverted orientation. The upright face on the right is the same as the inverted one. It is presented here for the sake of comparison. 


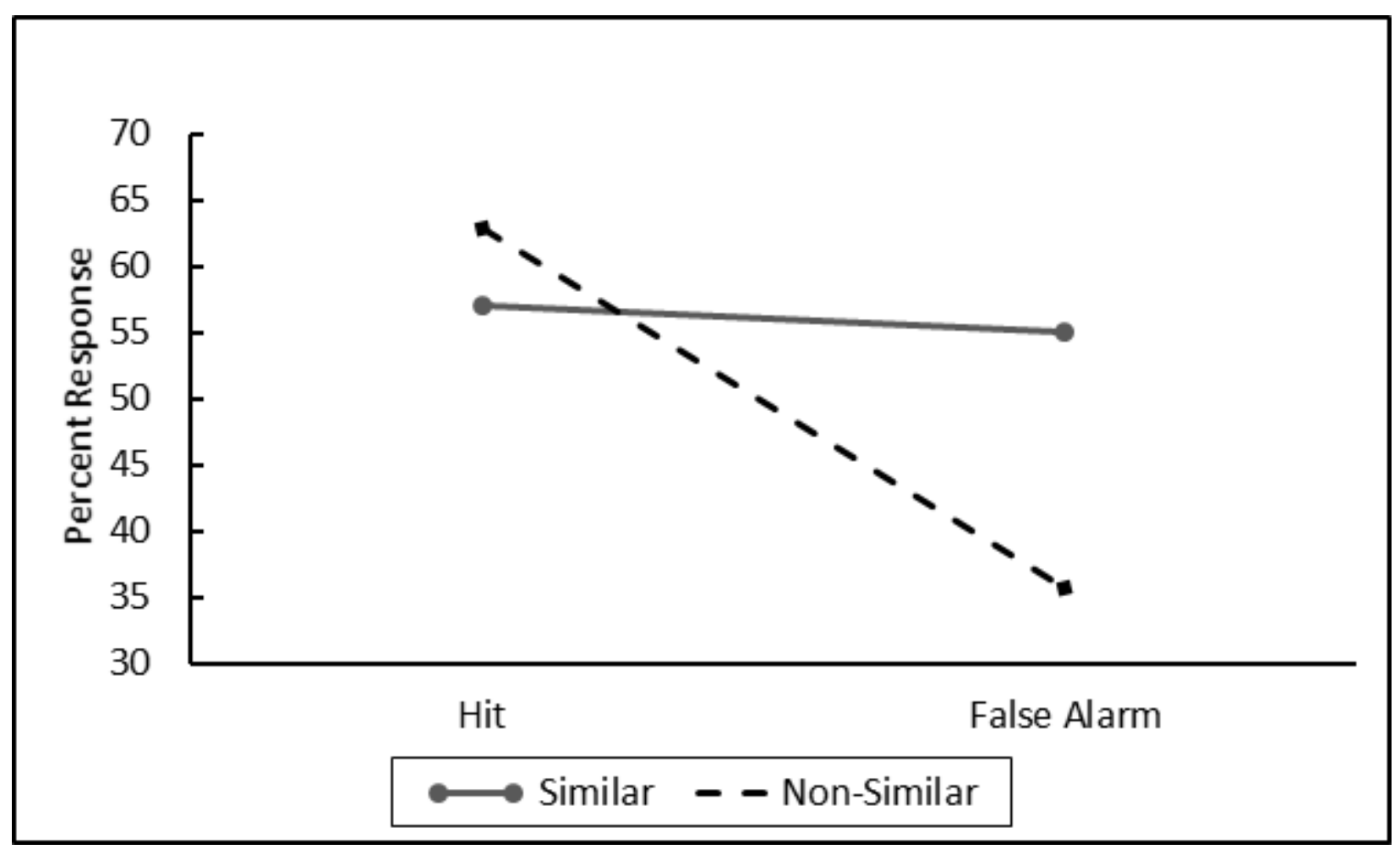

\section{Figure 2}

Percent Hits and False Alarm as function of similar and non-similar groups of pairs of different ovalfaces.

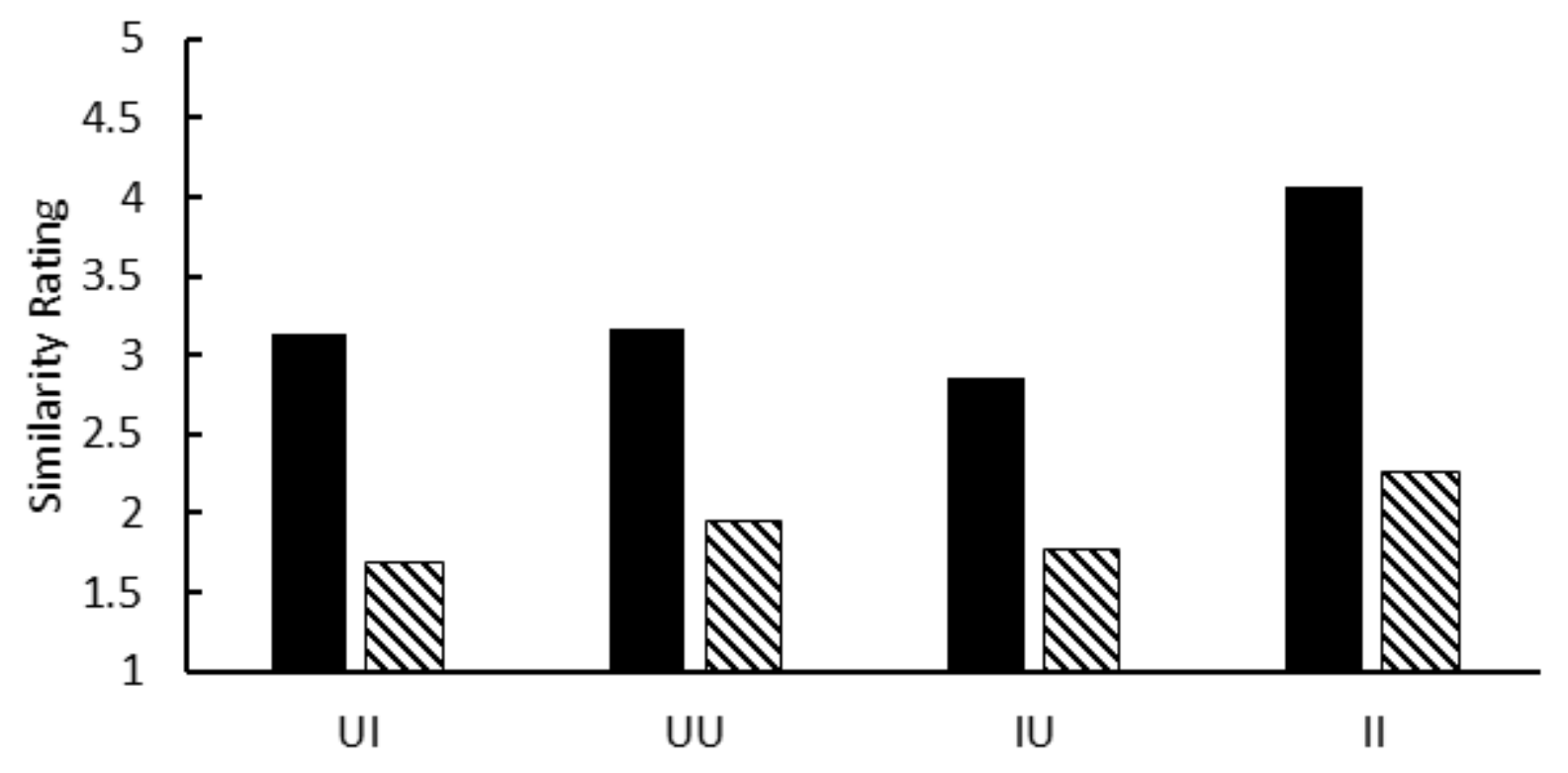

Similar $\mathbf{\Delta}$ Non-Similar

\section{Figure 3}

Similarity ratings as a function of the face pair orientation and of similarity versus non-similarity. 